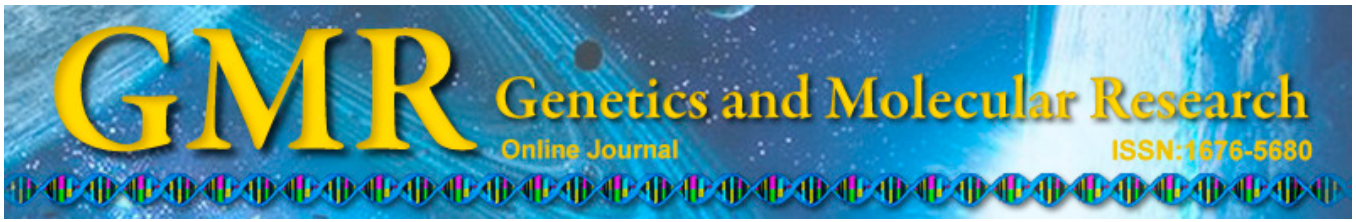

\title{
Superoxide radical-generating compounds activate a predicted promoter site for paraquat-inducible genes of the Chromobacterium violaceum bacterium in a dose-dependent manner
}

J.E. Gabriel ${ }^{1}$, E.P. Guerra-Slompo ${ }^{2}$, E.M. de Souza ${ }^{3}$, F.A.L. de Carvalho ${ }^{1}$, H.M.F. Madeira ${ }^{2}$ and A.T.R. de Vasconcelos ${ }^{4}$

${ }^{1}$ Colegiado de Ciências Biológicas, Centro de Ciências Agrárias, Universidade Federal do Vale do São Francisco, Petrolina, PE, Brasil ${ }^{2}$ Campus de Ciências Agrárias, Pontifícia Universidade Católica do Paraná, São José dos Pinhais, PR, Brasil ${ }^{3}$ Setor de Ciências Biológicas, Departamento de Bioquímica, Universidade Federal do Paraná, Curitiba, PR, Brasil

${ }^{4}$ Laboratório Nacional de Computação Científica, Petrópolis, RJ, Brasil

Corresponding author: J.E. Gabriel

E-mail: jane.gabriel@univasf.edu.br

Genet. Mol. Res. 14 (3): 10139-10144 (2015)

Received December 18, 2014

Accepted May 27, 2015

Published August 21, 2015

DOI http://dx.doi.org/10.4238/2015.August.21.20

\begin{abstract}
The purpose of the present study was to functionally evaluate the influence of superoxide radical-generating compounds on the heterologous induction of a predicted promoter region of open reading frames for paraquat-inducible genes ( $p q i$ genes) revealed during genome annotation analyses of the Chromobacterium violaceum bacterium. A 388-bp fragment corresponding to a pqi gene promoter of C. violaceum was amplified using specific primers and cloned into a conjugative vector containing the Escherichia coli lacZ gene without a
\end{abstract}


promoter. Assessments of the expression of the $\beta$-galactosidase enzyme were performed in the presence of menadione (MEN) and phenazine methosulfate (PMS) compounds at different final concentrations to evaluate the heterologous activation of the predicted promoter region of interest in $C$. violaceum induced by these substrates. Under these experimental conditions, the MEN reagent promoted highly significant increases in the expression of the $\beta$-galactosidase enzyme modulated by activating the promoter region of the $p q i$ genes at all concentrations tested. On the other hand, significantly higher levels in the expression of the $\beta$-galactosidase enzyme were detected exclusively in the presence of the PMS reagent at a final concentration of $50 \mu \mathrm{g} / \mathrm{mL}$. The findings described in the present study demonstrate that superoxide radical-generating compounds can activate a predicted promoter DNA motif for $p q i$ genes of the $C$. violaceum bacterium in a dose-dependent manner.

Key words: Chromobacterium violaceum; Functional genome; Menadione; Paraquat-inducible genes; Phenazine methosulfate

\section{INTRODUCTION}

Chromobacterium violaceum is a Gram-negative, free-living beta-proteobacterium that dominates a variety of ecosystems in tropical and subtropical regions. Over the last decade, genome prospecting of this bacterium has revealed several refined mechanisms related to its remarkable and exploitable adaptability (Brazilian National Genome Project Consortium, 2003). Genome annotation analyses of $C$. violaceum have identified the presence of open reading frames (ORFs) with high similarity to sequences targeting paraquat-inducible genes ( $p q i$ genes) previously characterized in Escherichia coli (Farr and Kogama, 1991). The pgi genes are drastically modulated by the action of several oxidizing agents to minimize their deleterious effects on cellular homeostasis (Hungria et al., 2004). Bacterial cells respond to oxidative stress by inducing numerous regulons controlled by transcription factors responding either directly or indirectly to oxidizing agents.

Menadione (MEN) and phenazine methosulfate (PMS) substrates are characterized as important superoxide radical-generating compounds that generates reactive oxygen species through redox cycling, and high concentrations of these substrates trigger cell death (Loor et al., 2010). MEN has been implicated in the activation of the mitochondrial permeability transition pore as a mediator of cell death (Azevedo et al., 2014; Reed et al., 2014). Furthermore, PMS causes intracellular $\mathrm{Ca}^{2+}$ to rise and dramatically increases membrane permeability for $\mathrm{K}^{+}$by activating Gardos channels, which results in the dehydration of circulating red blood cells (Gibson et al., 2003; Shcherbachenko et al., 2007).

Within this perspective, the purpose of the present study was to functionally evaluate the influence of the MEN and PMS compounds on the heterologous induction of a predicted promoter region of ORFs for the pqi genes identified during genome annotation analyses of the $C$. violaceum bacterium. The characterization of the functional proprieties of this regulatory DNA motif might provide insights into the biological responses of differing bacterial strains to the gene activation likely involved in the molecular mechanisms of environmental adaptation to oxidizing agents. 


\section{MATERIAL AND METHODS}

\section{Amplification of the pqi promoter and generation of $l a c Z$ fusion constructs}

Assays to measure the expression of the $\beta$-galactosidase enzyme were carried out to evaluate the effects of superoxide radical-generating compounds on the predicted promoter region of the pqi genes of $C$. violaceum. First, specific primers were designed using a computational program PrimerQuest (Integrated DNA Technologies, http://www.idtdn.com/SciTools/SciTools.aspx) to amplify target sites situated between the ORFs CV2550 and CV2551 corresponding to the predicted promoter region of the $p q i$ genes of $C$. violaceum. Forward (5'CGT GAA TTC TAA TGG CAG ACC GAC ATC AG-3') and reverse (5'-GGT AGA TCT TTT CGT GCG GGT GCT GTT TC-3') primer sequences were constructed according to Sambrook and Russell (2001) to contain specific DNA cleavage sites for the restriction enzymes EcoRI and $B g l \mathrm{II}$ (underlined bases), respectively. Genomic DNA of $C$. violaceum isolated using a standard saline solution and phenol-chloroform extraction method was amplified in Applied Biosystems $^{\circledR} 2720$ Thermal Cycler (Life Technologies, Gaithersburg, MD, USA) in the presence of PCR buffer $1 \mathrm{X}(20 \mathrm{mM}$ Tris-HCl, $\mathrm{pH} 8.4,50 \mathrm{mM} \mathrm{KCl}), 0.4 \mathrm{mM}$ of each dNTPs, 2.5 $\mathrm{mM}$ magnesium chloride, $1.2 \mathrm{mM}$ specific primers, and $1 \mathrm{U}^{\text {Platinum }}{ }^{\circledR}$ Taq DNA Polymerase (Life Technologies) at a final volume of $25 \mathrm{~mL}$. The amplification reactions consisted of denaturation at $95^{\circ} \mathrm{C}$ for $30 \mathrm{~s}$, annealing at $64^{\circ} \mathrm{C}$ for $45 \mathrm{~s}$, and extension at $72^{\circ} \mathrm{C}$ for $45 \mathrm{~s}$, for a total of 35 cycles. The resulting 388-bp amplicon was ligated into the conjugative vector pMP220 previously digested with restriction enzymes EcoRI and BglII. This broad host range conjugative vector contained the E. coli lacZ gene without a promoter to generate transcriptional lac $Z$ gene fusions (Spaink et al., 1987). Competent E. coli $S 17$ strains were electrotransformed with the amplicon for insertion into the conjugative vector carrying the promoter region of interest, as established by Sambrook and Russel (2001), followed by cellular growth of the bacterial isolates on Luria-Bertani agar containing $12.5 \mathrm{mg} / \mathrm{mL}$ tetracycline at $37^{\circ} \mathrm{C}$.

\section{Analysis of pqi promoter activation via the lacZ reporter gene}

Heterologous induction of the promoter region of the pqi genes of C. violaceum was evaluated in the presence of superoxide radical-generating compounds by measuring the expression levels of the $\beta$-galactosidase enzyme in the presence of the orthonitrophenyl- $\beta$-D-galactopyranoside reagent (Sigma-Aldrich, St Louis, MO, USA), as proposed in detail by Miller (1972). The $\beta$-galactosidase enzyme expression assays were carried out using $100 \mu \mathrm{L}$ saturated culture of transformed $E$. coli cells diluted in $4.9 \mathrm{~mL}$ Luria Broth medium containing $12.5 \mu \mathrm{g} / \mathrm{mL}$ tetracycline and maintained at $37^{\circ} \mathrm{C}$ under aeration conditions. To achieve an $\mathrm{OD}_{600 \mathrm{~nm}}$ reading of 0.25 (approximately 2 -h incubation), MEN (2-methyl-1,4-naphthoquinone) and PMS (N-methyldibenzopyrazine bromide) compounds commercially acquired from Sigma-Aldrich were added to the bacterial inoculums at the final concentrations of 50 and $100 \mu \mathrm{g} / \mathrm{mL}$, with 5 -h additional incubation under same conditions to trigger the induction of the promoter region. Two distinct $E$. coli isolates carrying promoter-lac $Z$ gene fusions were designed pqi promoters 12 and 13 and were selected and tested. Alternatively, E. coli strains carrying pqi promoters 12 and 13 were maintained under the same experimental conditions without addition of the compounds, constituting the control groups. 


\section{Statistical analysis}

Descriptive statistical analyses of the data were performed using the software STATISTICA/W statistical package version 10.0 (Statsoft, Tulsa, OK, USA) from three independent experimental assays. Results were presented as means, standard deviations, and standard errors of the means and were estimated by using hierarchical linear model and analysis of variance, with a $\mathrm{P}$ value $<0.05$ considered to be statistically significant.

\section{RESULTS}

The heterologous induction of a predicted promoter region for the pqi genes of the C. violaceum bacterium was evaluated by measuring the expression levels of $\beta$-galactosidase in the presence of different superoxide radical-generating compounds, as shown in Figure 1. The MEN reagent promoted a highly significant increase in the expression of $\beta$-galactosidase modulated by activating the promoter region of the $p q i$ genes of $C$. violaceum, independent of concentration $(\mathrm{P}<0.05)$, with 2 -fold higher response to MEN than under control conditions (Figure 1A). In contrast, the induction of the pqi gene promoter was drastically affected by changing the final concentration of the PMS reagent, as significant increases in $\beta$-galactosidase expression in the presence of the PMS reagent were detected exclusively at a final concentration of $50 \mu \mathrm{g} / \mathrm{mL}(\mathrm{P}<0.05)$ (Figure 1B). No significant differences in the expression levels of the $\beta$-galactosidase enzyme were observed in the presence of the PMS reagent at a final concentration of $100 \mu \mathrm{g} / \mathrm{mL}(\mathrm{P}=0.152)$ (Figure 1B).
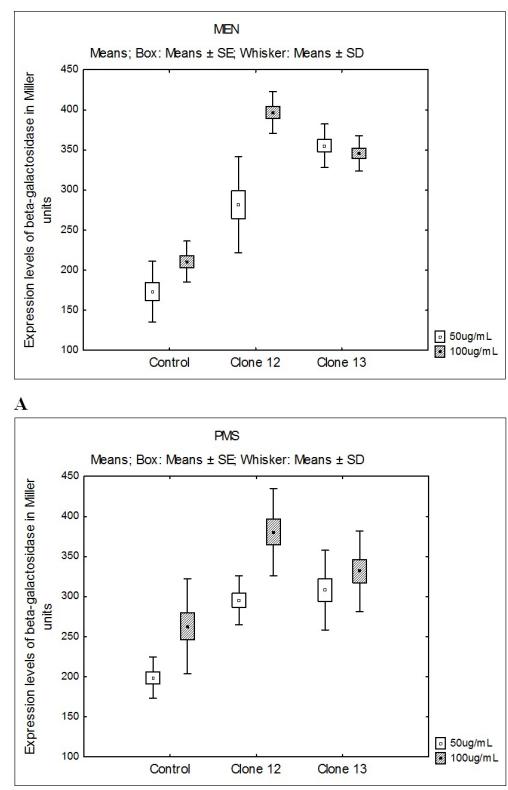

Figure 1. Expression levels of the $\beta$-galactosidase enzyme in response to activation of predicted promoter regions for paraquat-inducible genes of Chromobacterium violaceum exposed to MEN (A) or PMS (B) at final concentrations of 50 and $100 \mu \mathrm{g} / \mathrm{mL}$. Results are reported as means, standard deviations (SD), and standard errors of the means (SE). 
In addition, to verify the eventual intrinsic resistance of the $C$. violaceum bacterium in the presence of the compounds tested, colony-forming units (CFUs) per milliliter were calculated by monitoring the growth of the $C$. violaceum colonies on LB agar plates containing MEN and PMS compounds at final concentrations of 0 (control conditions), 25, 40, 60, $75,100,1000$, and $2800 \mu \mathrm{g} / \mathrm{mL}$. Bacterial growth of $C$. violaceum colonies was exclusively detected in the presence of MEN at a final concentration of $25 \mu \mathrm{g} / \mathrm{mL}\left(10^{5} \mathrm{CFU} / \mathrm{mL}\right)$. On the other hand, no CFUs were detected in response to PMS at any concentrations tested. Approximately $10^{7} \mathrm{CFU} / \mathrm{mL}$ of the $C$. violaceum bacterium were obtained under control conditions of bacterial growth on agar plates without addition of either compound.

\section{DISCUSSION}

The findings described herein demonstrate that different superoxide radical-generating compounds could induce a putative promoter region for the pqi genes of the $C$. violaceum bacterium in a dose-dependent manner (Figure 1). Recently, researchers have examined the heterologous activation of regulatory DNA motifs of the $C$. violaceum bacterium in response to superoxide radical-generating substrates. Within this perspective, Gabriel et al. (2015) have reported that the paraquat compound itself provoked significant increases in the expression levels of the $\beta$-galactosidase enzyme in $E$. coli strains carrying the predicted promoter sequence for the $p q i$ genes of $C$. violaceum fused to the $l a c Z$ gene; in this study, reported values were 3.5 to 4 -fold higher in response to paraquat than were those observed in the control group. These results suggested the intrinsic existence of regulatory DNA motifs in the genome of $C$. violaceum that would be potentially inducible by an oxidizing agent (Gabriel et al., 2015).

Over the past decades, some studies have examined the molecular mechanisms involved in the gene activation associated with the remarkable cellular response to environment adaptation seen in the C. violaceum bacterium (Hungria et al., 2004; Baraúna et al., 2011; Ciprandi et al., 2012). In fact, the free-living microorganism C. violaceum is exposed frequently to a series of abiotic factors such as different sources and abundance of nutrients, changes in temperature and $\mathrm{pH}$, as well as toxic compounds and UV rays. The molecular characterization in this study of a pqi gene promoter sequence detected in the $C$. violaceum genome that is activated by different superoxide radical-generating substrates seems to provide supporting evidence for the great adaptability to a wide range of environments and strong protective systems triggered within these bacterial cells.

Furthermore, this study also clarified experimentally the functional properties of a regulatory DNA motif predicted previously in ORFs for pqi genes during genome annotation of the $C$. violaceum bacterium (Figure 1). With the advent of the genomic era, innumerable advances have arisen in basic science research, and extensive applications in a great variety of fields such as genetics and environment adaptation have been conducted. According to Feder and Mitchell-Olds (2003), the detailed elucidation of molecular, cellular, and organismal mechanisms is frequently essential to understanding their adaptive physiological aspects and evolutionary impact.

In conclusion, the significant effects of the MEN and PMS reagents on the heterologous induction of a predicted promoter region for the pqi genes in the C. violaceum genome seems to provide evidence for the functional aspects of the influence of superoxide radicalgenerating compounds on the activation of a potential molecular response to adaptive environments in this bacterium. 


\section{Conflicts of interest}

The authors declare no conflict of interest.

\section{ACKNOWLEDGMENTS}

The authors are grateful to Ministério da Ciência e Tecnologia (MCT)/Conselho Nacional de Desenvolvimento Científico e Tecnológico (CNPq) for financial support.

\section{REFERENCES}

Azevedo RF, Souza RK, Braga GU and Rangel DE (2014). Responsiveness of entomopathogenic fungi to menadioneinduced oxidative stress. Fungal Biol. 118: 990-995.

Baraúna RA, Ciprandi A, Santos AV, Carepo MS, et al. (2011). Proteomics analysis of the effects of cyanate on Chromobacterium violaceum metabolism. Genes 2: 736-747.

Brazilian National Genome Project Consortium (2003). The complete genome sequence of Chromobacterium violaceum reveals remarkable and exploitable bacterial adaptability. Proc. Natl. Acad. Sci. U. S. A. 100: 11660-11665.

Ciprandi A, Baraúna RA, Santos AV, Gonçalves EC, et al. (2012). Proteomic response to arsenic stress in Chromobacterium violaceum. J. Int. OMICS 2: 69-73.

Farr SB and Kogama T (1991). Oxidative stress responses in Escherichia coli and Salmonella typhimurium. Microbiol. Rev. 55: 561-585.

Feder ME and Mitchell-Olds T (2003). Evolutionary and ecological functional genomics. Nature Rev. Genet. 4: 651-657.

Gabriel JE, Guerra-Slompo EP, de Carvalho FAL, Madeira HMF, et al. (2015). Heterologous induction of a predicted promoter sequence for paraquat-inducible genes of Chromobacterium violaceum in response to paraquat compound. Braz. J. Biol. 75: 503-504.

Gibson JS, Muzymba MC and Ellory CJ (2003). Effect of phenazine methosulfate on K+ transport in human red cells. Cell Physiol. Biochem. 13: 329-336.

Hungria M, Nicolás MF, Guimarães CT, Jardim SN, et al. (2004). Tolerance to stress and environmental adaptability of Chromobacterium violaceum. Genet. Mol. Res. 3: 102-116.

Loor G, Kondapalli J, Schriewer JM, Chandel NS, et al. (2010). Menadione triggers cell death through ROS-dependent mechanisms involving PARP activation without requiring apoptosis. Free Radic. Biol. Med. 49: 1925-1936.

Miller JH (1972). Assay of $\beta$-galactosidase, In Experiments in Molecular Genetics. CSH Laboratory Press, New York, 352-355.

Reed KN, Wilson G, Pearsall A and Grishko VI (2014). The role of mitochondrial reactive oxygen species in cartilage matrix destruction. Mol. Cell Biochem. 397: 195-201.

Sambrook J and Russel DW (2001). Molecular cloning: a laboratory manual. 3rd edn. Cold Spring Harbor, New York.

Shcherbachenko IM, Lisovskaya IL and Tikhonov VP (2007). Oxidation-induced calcium-dependent dehydration of normal human red blood cells. Free Radic. Res. 41: 536-545.

Spaink HP, Okker JH, Wijffelman CA, Pees E, et al. (1987). Promoters in the nodulation region of the Rhizobium leguminosarum Sym plasmid pRL1JL. Plant Mol. Biol. 9: 27-39. 